\title{
Preparation of Double Liposomes and Their Efficiency as an Oral Vaccine Carrier
}

\author{
Shinsuke Ogue ${ }^{a}$ Yuri Takahashi, ${ }^{*}, b$ Hiraku ONISHI,${ }^{a}$ and Yoshiharu Machida ${ }^{a}$ \\ ${ }^{a}$ Department of Drug Delivery Research, Hoshi University; and ${ }^{b}$ Research Center for Pharmaceutical Education, Hoshi \\ University; 2-4-41 Ebara, Shinagawa-ku, Tokyo 142-8501, Japan. \\ Received December 22, 2005; accepted March 13, 2006; published online March 17, 2006
}

\begin{abstract}
The usefulness of double liposomes (DL), liposomes containing liposomes inside, as an oral vaccine carrier was examined. Ovalbumin (OVA) encapsulating liposomes sized to $230 \mathrm{~nm}$ (small liposomes, SL) were prepared by the glass-beads (GB) method and sequential sonication and extrusion. For the purpose of stabilizing the model antigen, DL containing SL were prepared by the GB method and the reverse-phase evaporation (REV) method. They were named GB-DL and REV-DL, respectively. The morphological structure of DL was confirmed using confocal laser scanning microscopy and scanning electron microscopy by the freeze-fracture method. DL showed suppressed release of OVA and stabilized OVA in pepsin solution as compared with SL. BALB/c mice were immunized with OVA solution, SL and DL suspension by oral administration. Significantly higher levels of IgA in feces were observed in mice immunized with SL and REV-DL as compared with OVA solution, and REVDL tended to show the higher level of IgA than SL. REV-DL elicited significantly higher anti-OVA IgG responses as compared with OVA solution. Furthermore, GB-DL tended to raise the IgG level as compared with SL. The results suggest that DL have the potential to be an effective carrier for oral immunization.
\end{abstract}

Key words double liposome; oral vaccine; ovalbumin; glass-beads; mucosal immunity

Vaccination is one of the best preventive strategies against infectious disease. Most of the vaccines available today are injected parenterally, and they are not effective for inducing immunity at mucosal surfaces. In contrast, oral vaccines elicit both mucosal and systemic immune responses and have the advantages of self-administration. However, the oral administration of vaccines has the problem of antigen degradation by gastric acid and proteolytic enzymes in the digestive system, and extremely large doses are required to achieve an adequate immune response. Therefore, the use of particles such as liposomes as a carrier to prevent the degradation of antigens has been investigated. ${ }^{1-6)}$ Liposomes are attractive as an antigen delivery carrier since their particle size, surface charge and membrane fluidity can be easily controlled. Besides, the natural tendency of liposomes to interact with macrophages has served as the primary rationale for utilizing liposomes as a carrier of antigens. ${ }^{7)}$ However, usual liposomes can be rapidly degraded by bile salts and other components of the gastrointestinal intraluminal environment. Therefore, stabilized liposomes such as polysaccharidecoated liposomes and polymerized liposomes have been investigated. ${ }^{8-10)}$

In previous studies, double liposomes (DL), in which several small liposomes (SL) were encapsulated in a large liposome, were prepared by a glass-filter method and a glassbeads (GB) method. ${ }^{11,12)}$ Salmon calcitonin- and insulinloaded DL exerted good hypocalcemic and hypoglycemic effects via oral administration, respectively. ${ }^{13-15)}$ We suggest that the use of DL as a vaccine career will be advantageous for improving the protection of the antigen. That is, it is hypothesized regarding the concept of vaccine delivery using DL that the outer liposomes are degraded by lipase, bile salts etc. after protecting the inner liposomes until they reach the lower small intestine, and then SL are taken up by the M cells in the intestinal lumen. The strength of the bilayer can be adjusted by changing the lipid compositions of SL and the outer lipid.
In the present study, we prepared SL containing ovalbumin (OVA) as a model antigen by the GB method, and then obtained DL by encapsulation of SL in a large liposome by the GB method or the reverse-phase evaporation (REV) method. ${ }^{16,17)}$ SL were sized to a desired particle diameter to be encapsulated in a large liposome by sequential sonication and extrusion. The morphological structure of DL was examined using confocal laser scanning microscopy and scanning electron microscopy by the freeze-fracture method. In vitro characteristics of SL and DL were determined. Furthermore, systemic and mucosal immune responses following oral administration of the liposomes to mice were examined.

\section{MATERIALS AND METHODS}

Materials Hydrogenated soybean phosphatidylcholine (SoyPC) was kindly supplied by NOF Co. Ltd. (Tokyo, Japan). Dimyristoyl-phosphatidylcholine (DMPC) and dimyristoyl-phosphatidylglycerol (DMPG) were purchased from Sigma Chemical Company (St. Louis, U.S.A.). Dipalmitoyl-phosphatidylcholine (DPPC), cholesterol (Ch), stearylamine $(\mathrm{SA})$ and OVA $(5 \times$ crystalline) were purchased from Wako Pure Chemical Industries, Ltd. (Osaka, Japan). The BCA Protein Assay Reagent kit was obtained from Pierce Biotechnology, Inc. (IL, U.S.A.). The Mouse IgA ELISA Quantitation Kit and Mouse IgG ELISA Quantitation Kit were obtained from Bethyl Laboratories, Inc. (TX, U.S.A.). The TMB Microwell Peroxidase Substrate System was obtained from Kirkegaard \& Perry Laboratories, Inc. (MD, U.S.A.). Glass beads (BZ-2: $1.5-2.5 \mathrm{~mm}$ diameter) were purchased from As One (Osaka, Japan). All other chemicals and solvents were obtained commercially as reagent grade products.

Animals Female BALB/c mice (8-week-old) were purchased from Charles River (Yokohama, Japan). They were housed in the specific pathogen-free (SPF) conditions, and used soon after purchase. All experiments in the present 
study conformed to the Guidelines for Animal Experimentation of Hoshi University.

Preparation of Inner Liposomes The inner liposomes (SL) were prepared by the GB method. ${ }^{12)}$ Namely, a lipid mixture containing SoyPC, DPPC, $\mathrm{Ch}$ and SA in a molar ratio of $7: 7: 5: 4$ (total amount, $52 \mu \mathrm{mol}$ ) was dissolved in chloroform. This solution was poured into a Kjeldahl flask with glass beads. The organic solvent was evaporated using a rotary evaporator for $1 \mathrm{~h}$ and the residual organic solvent was removed under vacuum overnight. At $55^{\circ} \mathrm{C}, 1.5 \mathrm{ml}$ of phosphate buffered saline (PBS, $\mathrm{pH}$ 7.4) containing OVA $(8 \mathrm{mg} / \mathrm{ml})$ was added to the Kjeldahl flask and incubated for $15 \mathrm{~min}$. After vigorous agitation for $10 \mathrm{~min}$, the resultant liposomes were separated from the glass beads by aspiratory filtration. The liposome suspension was centrifuged three times at $5000 \mathrm{rpm}$ for $10 \mathrm{~min}$ to remove untrapped OVA, and the residue was resuspended in PBS. Then, the suspension was sonicated for $10 \mathrm{~min}$ at $55^{\circ} \mathrm{C}$, and passed through a 0.8 $\mu \mathrm{m}$ polycarbonate membrane using an Avanti Mini-extruder (Avanti Polar Lipids, AL, U.S.A.) until a uniform liposome size was achieved. After ultracentrifugation at $24000 \mathrm{rpm}$ for $20 \mathrm{~min}$ to remove untrapped OVA, the residue was resuspended in PBS to obtain the SL suspension. To measure the encapsulation efficiency, one-fourth of the SL suspension prepared was used.

Preparation of Double Liposomes by the Glass-Beads Method To form the outer lipid layer for DL, a lipid mixture containing DMPC and DMPG at a molar ratio of $10: 1$ (total amount, $39 \mu \mathrm{mol}$ ) dissolved in chloroform was poured into the Kjeldahl flask with glass beads. The organic solvent was evaporated using a rotary evaporator for $1 \mathrm{~h}$, and then the residual organic solvent was removed under vacuum overnight. The lipid layer formed on glass beads was hydrated with the SL suspension described above for $15 \mathrm{~min}$ at $35^{\circ} \mathrm{C}$. After vigorous agitation for $10 \mathrm{~min}$, the resultant liposomes were separated from the glass beads by aspiratory filtration. The liposome suspension was centrifuged at 3000 rpm for $10 \mathrm{~min}$, and the supernatant was removed. Then, the suspension of DL prepared by the GB method (GB-DL) was obtained by resuspending the residue in PBS.

Preparation of Double Liposomes by the Reverse-Phase Evaporation Method A lipid mixture containing DMPC and DMPG at a molar ratio of 10:1 (total amount, $39 \mu \mathrm{mol}$ ) dissolved in chloroform was poured into a Kjeldahl flask, and deposited on the flask by removal of the organic solvent by rotary evaporation. The lipid was then redissolved in $3 \mathrm{ml}$ of chloroform, and the SL suspension described above was added. The mixture was sonicated for $5 \mathrm{~min}$ at $20^{\circ} \mathrm{C}$ to form a w/o emulsion, and the organic solvent was slowly removed by rotary evaporation until the suspension became a gel, followed by brief vortex mixing. Then the residual organic solvent was evaporated until a homogeneous suspension was obtained. The resultant liposomal suspension was centrifuged at $3000 \mathrm{rpm}$ for $10 \mathrm{~min}$, and the supernatant was removed. Then, the suspension of DL prepared by the REV method (REV-DL) was obtained by resuspending the residue in PBS.

Morphological Investigation of Double Liposomes Morphological structures of GB-DL and REV-DL were investigated using confocal laser scanning microscopy (Radiance 2100, Nippon Bio-Rad Laboratories) and scanning elec- tron microscopy (JM-1010, JEOL Ltd.) by the freeze-fracture method. For investigations using confocal laser scanning microscopy, SL containing FITC-labeled OVA (FITC-OVA) instead of OVA were prepared, and GB-DL and REV-DL were prepared by encapsulation of the SL using outer lipids containing 1,1'-dioctadecyl-3,3,3',3'-tetra-methylindocarbocyanine perchlorate (DiI).

Encapsulation Efficiency and DL Formative Efficiency For examination of encapsulation efficiency, $0.5 \mathrm{ml}$ of $10 \%$ Triton X-100 was added to the SL suspension to destroy the liposomes, and the amount of OVA released was measured using the BCA Protein Assay Kit. The encapsulation efficiency $(\%)$ was calculated using the following equation:

encapsulation efficiency $(\%)=($ amount of OVA encapsulated in SL/amount of OVA used) $\times 100$

Similarly, the above procedure was performed for DL, and DL formative efficiency (\%) was calculated using the efficiencies of encapsulation in SL and DL as follows:

DL formative efficiency $(\%)=($ efficiency of encapsulation in DL/ efficiency of encapsulation in SL) $\times 100$

Release Properties and Stability The liposome suspensions prepared (SL, GB-DL and REV-DL) were centrifuged at $24000 \mathrm{rpm}$ for $20 \mathrm{~min}$, and the supernatant was removed. The precipitate was resuspended in $4 \mathrm{ml}$ of the first fluid ( $\mathrm{pH}$ 1.2) in Japanese Pharmacopoeia XIV (JP XIV). After incubation with shaking at 100 strokes/min for $1 \mathrm{~h}$ at $37^{\circ} \mathrm{C}$, the suspensions were centrifuged, and the amounts of OVA released in the supernatant were measured using the BCA Protein Assay Kit. Then, the precipitate was resuspended in 10 $\mathrm{ml}$ of the second fluid ( $\mathrm{pH}$ 6.8) in JP XIV, and incubated with shaking at 100 strokes/min for $2 \mathrm{~h}$ at $37^{\circ} \mathrm{C}$. Periodically, 1-ml aliquots of the suspension were taken, and the sample solutions were analyzed as described above.

The stability of liposomes was also examined in pepsin solution. After centrifugation of liposome suspensions, the precipitate was resuspended in $5 \mathrm{ml}$ of pepsin solution (in $0.1 \mathrm{~mol} / 1$ glycine buffer, $\mathrm{pH} 1.3,5.0 \mathrm{U} / \mathrm{ml})$. The sample suspension was divided equally into three test tubes, and incubated with shaking at 100 strokes $/ \mathrm{min}$ at $37^{\circ} \mathrm{C}$ for 30,60 or $120 \mathrm{~min}$. After centrifugation, $10 \%$ Triton X-100 $(0.2 \mathrm{ml})$ was added to the precipitate. The amounts of OVA in the precipitate were measured as described above.

Immunization Female $\mathrm{BALB} / \mathrm{c}$ mice were starved overnight and administered $100 \mu \mathrm{l}$ of OVA solution in PBS $(1 \mu \mathrm{g} / \mu \mathrm{l})$ or liposome suspensions orally by gastric intubation. SL, GB-DL and REV-DL suspensions were diluted with PBS to adjust the content of OVA to $1 \mu \mathrm{g} / \mu \mathrm{l}$. The immunization was done twice with a 1 -week interval. Plasma was collected $7 \mathrm{~d}$ after the second immunization. Feces, which had been excreted for $24 \mathrm{~h}$ just before $7 \mathrm{~d}$ after the second immunization, were collected. After mixing the feces with PBS, the suspension was centrifuged and the supernatant was used as fecal extract. Immunological responses in the plasma and the fecal extracts were determined by ELISA. ${ }^{4,18)}$

The total amounts of IgA in the fecal extracts were measured using a mouse IgA ELISA Quantitation Kit. ${ }^{19)}$ The antiOVA IgG level in the plasma was measured by a modification of the method used with the mouse IgG ELISA Quantitation Kit as follows. ELISA plates were coated with $100 \mu \mathrm{l}$ of 
$50 \mathrm{mmol} / 1$ carbonate-bicarbonate buffer containing $10 \mu \mathrm{g} / \mathrm{ml}$ OVA ( $\mathrm{pH}$ 9.6). The plates were incubated overnight at $4{ }^{\circ} \mathrm{C}$, and then washed three times with wash solution $(50 \mathrm{mmol} / \mathrm{l}$ Tris, $0.14 \mathrm{~mol} / 1 \mathrm{NaCl}, 0.05 \%$ Tween 20, $\mathrm{pH} 8.0$ ). After the addition of $200 \mu \mathrm{l}$ of blocking solution $(50 \mathrm{mmol} / 1$ Tris, $0.14 \mathrm{~mol} / 1 \mathrm{NaCl}, 1 \% \mathrm{BSA}, \mathrm{pH} 8.0$ ), the plates were incubated for $1 \mathrm{~h}$ at $37^{\circ} \mathrm{C}$, and then washed three times. Then, $100 \mu \mathrm{l}$ of diluted samples were added to each well, and incubated for $2 \mathrm{~h}$ at $37^{\circ} \mathrm{C}$. After washing four times, $100 \mu \mathrm{l}$ of the substrate solution prepared according to the instructions for the TMB Microwell Peroxidase Substrate System was added, and incubated for $30 \mathrm{~min}$ at room temperature. To stop the TMB reaction, $100 \mu \mathrm{l}$ of $1 \mathrm{~mol} / 1$ phosphoric acid was applied to each well, and the absorbance at $450 \mathrm{~nm}$ was determined immediately with a microplate reader (Immuno Mini NJ-2300, Nalge Nunc International K.K.).

Statistical Analysis Variance in a group was evaluated by the $F$-test, and differences in immunological responses were evaluated by Student's $t$-test. The data were considered to be significantly different when the $p$-value was less than 0.05 .

\section{RESULTS AND DISCUSSION}

Characteristics of Small Liposomes Since the GB method resulted in high drug loading and high DL formation of liposomes in a previous study, ${ }^{12}$ ) we employed the GB method to prepare SL. The phase transition temperature of the lipids forming SL should be higher than that of the outer lipids. Therefore, SoyPC (Tc: $53^{\circ} \mathrm{C}$ ) and DPPC (Tc: $\left.41^{\circ} \mathrm{C}\right),{ }^{3,9)}$ with relatively high $\mathrm{Tc}$, were used as lipids forming SL. As stable liposomes are advantageous for preparing DL and for being taken up by the $\mathrm{M}$ cells as liposomes, $\mathrm{Ch}$, which has a stabilizing effect on the lipid bilayer, ${ }^{20}$ was added. In a preliminary experiment, high encapsulation efficiency of ovalbumin was observed when the lipids including a cationic lipid were used. Therefore, SA, with cationic charge, was also added. Characteristics of the SL prepared are shown in Table 1. The average particle size measured using an electrophoretic light scattering spectrophotometer (ELS-800, Otsuka Electronics Co., Japan) was $236 \mathrm{~nm}$. This diameter is considered appropriate for being enclosed by outer liposomes and taken up by the $\mathrm{M}$ cells. The $\zeta$-potential was positive owing to the addition of SA. It has been reported that the efficiency of encapsulation of hydrophilic antigens by liposomes is low. ${ }^{21-23)}$ However, the efficiency of encapsulation of OVA in SL was relatively high, $28.8 \%$.

Confirmation of DL and DL Formative Efficiency The phase transition temperature of the outer lipids should be lower than that of the inner lipids because SL might be damaged while preparing DL. Therefore, DMPC $\left(\mathrm{Tc}: 23^{\circ} \mathrm{C}\right)$ was used as the outer lipid. In a preliminary experiment, DL was prepared reproducibly when outer lipids including an anionic lipid were used. Therefore, DMPG, which has a negative charge, was added to DMPC. Although the GB method is simple and rapid, the obtained liposomes are usually multilamellar vesicles (MLV) that have a small interior space. Therefore, the REV method, which was developed to produce large uni- and oligolamellar vesicles, was also attempted. The images of GB-DL and REV-DL obtained by confocal laser scanning microscopy and scanning electron
Table 1. Characteristics of Small Liposomes and Double Liposomes

\begin{tabular}{lccc}
\hline \hline & $\begin{array}{c}\zeta \text {-potential } \\
(\mathrm{mV})\end{array}$ & $\begin{array}{c}\text { Encapsulation }^{c)} \\
\text { efficiency }(\%)\end{array}$ & $\begin{array}{c}\text { DL formative } \\
\text { efficiency }(\%)\end{array}$ \\
\hline SL & $42.6 \pm 3.4$ & $28.8 \pm 3.8$ & - \\
GB-DL & $-11.2 \pm 1.3$ & $24.9 \pm 2.2$ & $85.1 \pm 8.2$ \\
REV-DL & $7.2 \pm 3.6$ & $25.2 \pm 2.4$ & $94.0 \pm 4.0$ \\
\hline
\end{tabular}

a) $\zeta$-potential values $(n=3)$ are the mean of two independent preparations. $b$ ) Measured by ELS-800, Otsuka Electronics, Osaka, Japan. c) Each value represents the mean \pm S.D. $(n=4)$

microscopy are shown in Figs. 1-3. FITC-OVA incorporated in SL and outer liposomes can be distinguished in confocal microscopic images, but the detailed forms of the DL cannot be ascertained. In contrast, freeze-fracture electron micrographs show the detailed forms of DL, but cannot distinguish the inner lipid and the outer lipid of DL. Thus, observation with two kinds of microscopes is needed to definitely visualize the structure of DL. The green fluorescence of Fig. 1A represents FITC-OVA, and the red fluorescence of Fig. 1B represents DiI incorporated in the outer liposomes of GB-DL. From the combined image (Fig. 1C), it is confirmed that the green fluorescence is surrounded by the red fluorescence. However, outer liposomes without SL are also observed. Figure $3 \mathrm{~A}$ shows approximately $2.0-\mu \mathrm{m}$ GB-DL incorporating $100-400-\mathrm{nm}$ SL. Similarly, in the combined image of REV-DL (Fig. 2C), green fluorescence surrounded by red fluorescence is observed. The green spots of REV-DL are larger and there are fewer outer liposomes without SL, as compared with GB-DL. Figure 3B shows approximately 2.0$\mu \mathrm{m}$ REV-DL incorporating approximately 200-nm SL.

The characteristics of DL are shown in Table 1. Though the particle size of GB-DL was widely deviated from $1-$ $10 \mu \mathrm{m}$, the preparation of uniform-sized particles in the GB method was difficult. The particle size range of REV-DL was approximately $1-5 \mu \mathrm{m}$, which was narrower than that of GB-DL. The $\zeta$-potential of DL was decreased due to the influence of the anionic lipid of the outer liposomes. The $\zeta$-potential of GB-DL was lower than that of REV-DL. This may have been because more outer liposomes without SL were present in the GB-DL suspension. The DL formative efficiency of GB-DL and REV-DL was $85.1 \%$ and $94.0 \%$, respectively, which were relatively high values. However, there is some possibility that the OVA incorporated in coagulates of SL and the outer liposomes was counted when estimating the encapsulation efficiency of DL.

Release Properties and Stability Profiles of the release of OVA from liposomes are shown in Fig. 4. OVA was released gradually from SL, and the amount of OVA released at $180 \mathrm{~min}$ was $15.8 \%$. The rate of release of OVA from SL was almost stable regardless of the $\mathrm{pH}$ of the test solutions. Furthermore, GB-DL and REV-DL showed suppressed release of OVA as compared with SL. These findings suggested that OVA release was suppressed by double liposome formation and REV-DL were more effective than BG-DL for suppression of the release.

The stability of liposome-incorporated OVA against pepsin is shown in Fig. 5. Approximately 70\% of the OVA incorporated in SL was degraded at $120 \mathrm{~min}$. However, the OVA incorporated in GB-DL and REV-DL showed suppressed degradation during the examination period. Though the re- 
(A)

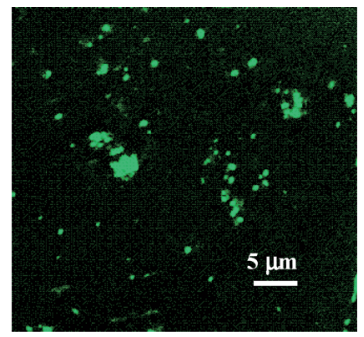

(B)

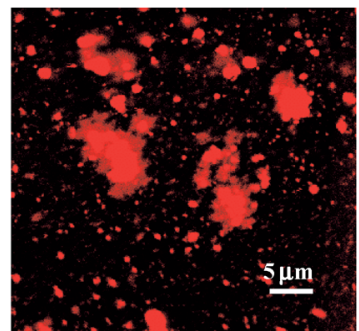

(C)

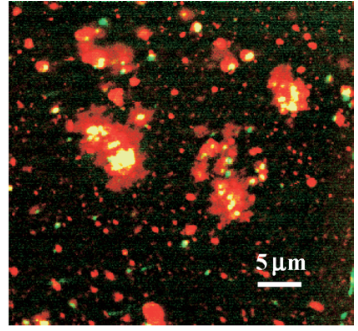

Fig. 1. Confocal Microscopic Images of GB-DL; FITC-OVA in Small Liposomes (A), DiI in Outer Lipids (B), Combined Image (C)

(A)

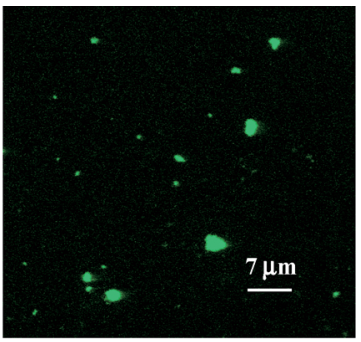

(B)

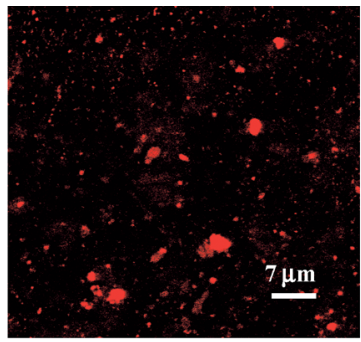

(C)

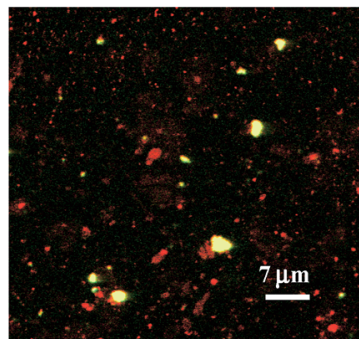

Fig. 2. Confocal Microscopic Images of REV-DL; FITC-OVA in Small Liposomes (A), DiI in Outer Lipids (B), Combined Image (C)

(A)

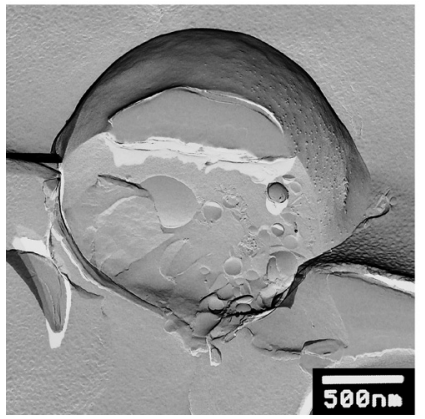

Fig. 3. Freeze-Fracture Electron Micrographs of GB-DL (A) and REV-DL (B)

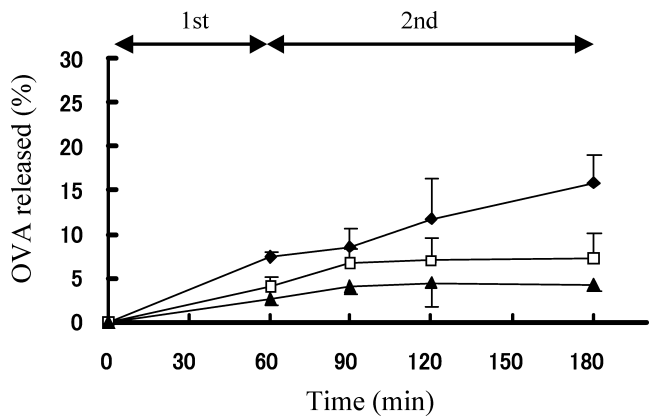

Fig. 4. Profiles of Release of OVA from Liposomes in the 2nd Fluid in JP XIV after Treatment in the 1st Fluid in JP XIV for $1 \mathrm{~h}$

Key: SL $(\diamond)$, GB-DL $(\square)$, REV-DL $(\boldsymbol{\Lambda})$. Each value represents the mean \pm S.D. $(n=3)$.

lease of OVA from liposomes was strongly suppressed in the release study, a large amount of OVA was degraded in the stability study. The reason for this may have been that glycine, which has the effect of destabilizing the lipid bilayer, ${ }^{24)}$ was used for the buffer solution. In this study, the protective effect of REV-DL against pepsin was higher than that of GB-DL. Unencapsulated SL might be more abundant in the GB-DL suspension. These results indicated that the
(B)
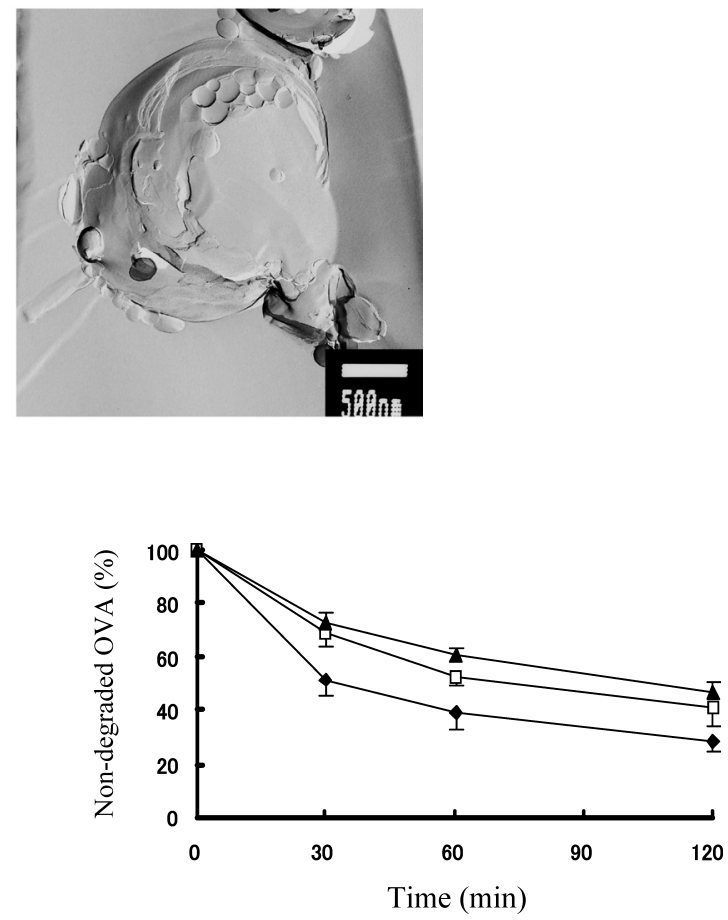

Fig. 5. Stability of OVA Encapsulated in Liposomes in Pepsin Solution ${ }^{a}$ )

Key: SL $(\diamond)$, GB-DL $(\square)$, REV-DL $(\boldsymbol{\Delta})$. Each value represents the mean \pm S.D. $(n=3)$. a) $5.0 \mathrm{U} / \mathrm{ml}$ pepsin in $0.1 \mathrm{~mol} / 1$ glycine buffer.

protective effect of liposomes on OVA was enhanced by the outer liposomes.

In Vivo Study The IgA responses in fecal extracts are shown in Fig. 6. The IgA level was significantly higher in SL and REV-DL suspensions than in OVA solution, and REVDL tended to show the higher IgA level than SL. The antiOVA IgG responses in plasma are shown in Fig. 7. The antiOVA IgG responses were compared using the ratio of absorbance between the animals administered OVA and the un- 


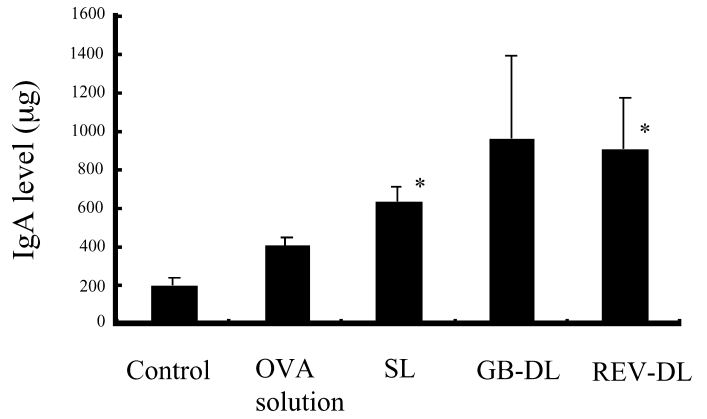

Fig. 6. Amount of IgA in Fecal Extracts Excreted for $24 \mathrm{~h}$ Just before $7 \mathrm{~d}$ after the Second Immunization

Each value represents the mean \pm S.D. $(n=3-4)$. $* p<0.05 v s$. OVA solution.

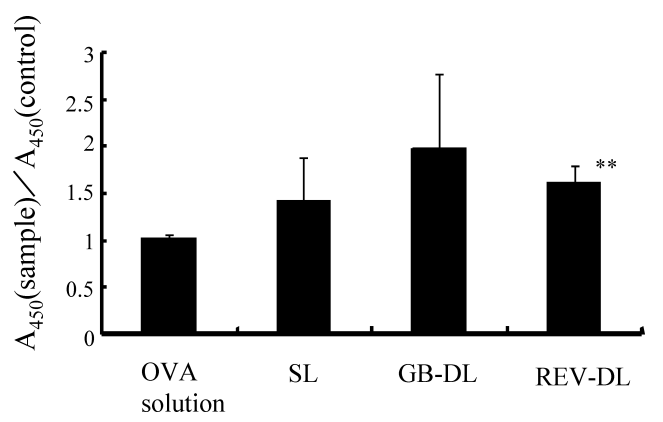

Fig. 7. Comparison of Anti-OVA IgG in Plasma $7 \mathrm{~d}$ after the Second Immunization

Each value represents the mean \pm S.D. $(n=4)$. $* * p<0.01 v s$. OVA solution.

treated animals (control). The $\mathrm{IgG}$ response was significantly greater in REV-DL suspensions than in OVA solution. Furthermore, GB-DL tended to raise the IgG level as compared with SL. In this study, a significant difference was not confirmed between SL and DL, because it was considered that the increase of the antibodies was moderate in every preparation by using OVA that was a relatively weak antigen. From these results, it was considered that liposomes could protect OVA from degradation in the gastrointestinal environments and elicit higher antibody responses than OVA solution. Since DL tended to exhibit higher antibody responses than $\mathrm{SL}$, it was considered that the stability of liposomes was an important factor for increasing the antibody responses. GBDL showed high antibody levels, but their variability was large. The reason for this was thought to be that the particle size of GB-DL, which was considered to be related to release of OVA or SL, uptake in Peyer's patches, was variable between each preparation. In order to prepare GB-DL more reproducibly, attempts to improve the method of preparation of GB-DL are in progress.

Since it was reported that particles smaller than $10 \mu \mathrm{m}$ were taken into Peyer's patches, ${ }^{2,21)}$ it is possible that intact DL is taken into Peyer's patches. However, we hypothesize that SL incorporated in DL is taken up after release from DL because the strength of the lipid bilayer of the outer liposomes is weak. To confirm this hypothesis, behavior of DL after oral administration should be investigated. Now, to clarify the uptake mechanism of OVA incorporated in liposomes, observation of Peyer's patches after oral administration of DL marked by fluorescent materials is in progress. Frey and Neutra $^{25}$ reported that hydrophobic or positively charged particle surfaces turned out to be best suited for $\mathrm{M}$ cell tar- geting via nonspecific interactions, because negatively charged mucus throughout the gut may act as a sink for positively charged particles. Since the SL prepared in this study had a positive charge, it is possible that they effectively interacted with $\mathrm{M}$ cells. Though the immunoadjuvant effect of liposomes for mucosal immunization has been studied by several groups, ${ }^{7,26)}$ a sufficient adjuvant effect for weak antigens was not reported. To enhance the immune responses, the use of adjuvants such as cholera toxin and lipopolysaccharide has been studied. ${ }^{18,23,27)}$ Since DL can incorporate an adjuvant in the interior space of outer liposomes and SL, increase of the immune responses by adding adjuvants may be expected. It is considered that the efficiency of DL as a vaccine carrier may become clearer by using adjuvants.

\section{CONCLUSIONS}

From the observation of DL using confocal laser scanning microscopy and scanning electron microscopy by the freezefracture method, the structure of SL incorporated by the outer liposomes was confirmed. The release of OVA from liposomes was very slow, and GB-DL and REV-DL showed a slower release rate than SL. GB-DL and REV-DL showed suppressed peptic degradation of OVA as compared with SL. The antibody responses were increased after oral administration of SL and DL as compared with OVA solution, and DL tended to elicit higher antibody responses than SL. These results suggest that DL could efficiently prevent the degradation of antigens after oral administration and function as a possibly useful oral vaccine carrier.

Acknowledgements This work was supported by the Ministry of Education, Culture, Sports, Science, and Technology of Japan. We appreciate the experimental assistance of Ms. Misato Akiya and Ms. Haruko Tsubouchi.

\section{REFERENCES}

1) Jani P., Halbert G. W., Langridge J., Florence A. T., J. Pharm. Pharmacol., 41, 809-812 (1989).

2) Uchida T., Goto S., Biol. Pharm. Bull., 17, 1272-1276 (1994).

3) Phillips N. C., Gagne L., Ivanoff N., Riveau G., Vaccine., 14, 898 904 (1996).

4) Kunisawa J., Okudaira A., Tsutusmi Y., Takahashi I., Nakanishi T., Kiyono H., Mayumi T., Vaccine., 19, 589-594 (2000).

5) Kim B., Bowersock T., Griebel P., Kidane A., Babiuk L. A., Sanchez M., Attah-Poku S., Kaushik R. S., Mutwiri G. K., J. Control. Release, 85, 191-202 (2002).

6) Minato S., Iwanaga K., Kakemi M., Yamashita S., Oku N., J. Control. Release, 89, 189-197 (2003).

7) Alving C. R., J. Immunol. Methods, 140, 1-13 (1991).

8) Chen H., Torchilin V., Langer R., Pharm. Res., 13, 1378-1383 (1996).

9) Han M., Watarai S., Kobayashi K., Yasuda T., J. Vet. Med. Sci., 59, 1109-1114 (1997).

10) Venkatesan N., Vyas S. P., Int. J. Pharm., 203, 169-177 (2000).

11) Katayama K., Kato Y., Onishi H., Nagai T., Machida Y., Int. J. Pharm., 248, 93-99 (2002).

12) Yamabe K., Kato Y., Onishi H., Machida Y., J. Control. Release, 90, $71-79$ (2003).

13) Ebato Y., Kato Y., Onishi H., Nagai T., Machida Y., Drug Dev. Res., 58, 253-257 (2003).

14) Katayama K., Kato Y., Onishi H., Nagai T., Machida Y., Drug Dev. Ind. Pharm., 29, 725-731 (2003).

15) Yamabe K., Kato Y., Onishi H., Machida Y., J. Control. Release, 89, $429-436$ (2003). 
16) Szoka F., Jr, Papahadjopoulos D., Proc. Natl. Acad. Sci. U.S.A., 75, 4194-4198 (1978).

17) Szoka F., Olson F., Heath T., Vail W., Mayhew E., Papahadjopoulos D., Biochim. Biophys. Acta, 601, 559-571 (1980).

18) Jackson R. J., Fujihashi K., Xu-Amano J., Kiyono H., Elson C. O., McGhee J. R., Infect. Immun., 61, 4272- 4279 (1993).

19) Nagamoto T., Hattori Y., Takayama K., Maitani Y., Pharm. Res., 21, $671-674$ (2004).

20) Vemuri S., Rhodes C. T., Pharm. Acta Helv., 70, 95-111 (1995).

21) Eldridge J. H., Meulbroek J. A., Staas J. K., Tice T. R., Gilley R. M., Adv. Exp. Med. Biol., 251, 191-202 (1989).
22) O'Hagan D. T., Palin K., Davis S. S., Artursson P., Sjoholm I., Vaccine., 7, 421-424 (1989).

23) Clarke C. J., Stokes C. R., Vet. Immunol. Immunopathol., 32, 139148 (1992).

24) Takahashi M., "Development of Functionality Cosmetics," CMC Publishing CO., LTD., Tokyo, 2000, p. 122.

25) Frey A., Neutra M. R., Behring Inst. Mitt., 98, 376-389 (1997).

26) Childers N. K., Michalek S. M., Pritchard D. G., McGhee J. R., Reg. Immunol., 3, 289-296 (1990).

27) Childers N. K., Miller K. L., Tong G., Llarena J. C., Greenway T., Ulrich J. T., Michalek S. M., Infect. Immun., 68, 5509-5516 (2000). 